\title{
Mental Health Outcomes Associated with Prenatal Alcohol Exposure: Genetic and Environmental Factors
}

\author{
Mary J. O’Connor
}

Published online: 17 June 2014

(C) Springer International Publishing Switzerland 2014

\begin{abstract}
Overwhelming evidence on the impact of maternal alcohol consumption during pregnancy has prompted increased attention to the link between prenatal alcohol exposure (PAE) and a constellation of developmental disabilities, which are characterized by physical, cognitive, and behavioral impairments. Importantly, individuals with PAE are particularly vulnerable to mental health problems. This review summarizes the current literature on the underlying mechanisms of PAE vulnerability, including epigenetic, genetic, and environmental risk factors that predispose individuals with PAE to psychiatric illness. The studies cited are from animal and human research and include a developmental perspective. Research on the mental health problems suffered by individuals with fetal alcohol spectrum disorders (FASDs) throughout development highlights the need for training of mental health professionals in the identification and the provision of specific treatments to address the unique features of this developmental disability.
\end{abstract}

Keywords Mental health · Psychiatric illness · Fetal alcohol spectrum disorders · Prenatal alcohol exposure · DSM 5 diagnosis

\section{Introduction}

Over the past 40 years, the teratogenic influence of prenatal alcohol exposure (PAE) on developmental outcomes has been extensively researched. Currently, individuals with PAE are described as having a wide range of disabilities characterized

\footnotetext{
M. J. O'Connor ( $\square)$

Department of Psychiatry and Biobehavioral Sciences, UCLA Semel Institute for Neuroscience and Human Behavior, David Geffen

School of Medicine at UCLA, 760 Westwood Plaza, Rm. 68-265A, Los Angeles, CA 90024, USA

e-mail: moconnor@mednet.ucla.edu
}

as fetal alcohol spectrum disorders (FASDs) [1 $\bullet$. Fetal alcohol syndrome (FAS), one of the most severe conditions resulting from in utero alcohol exposure, is defined by a pattern of characteristic growth deficiencies, facial malformations, and neurodevelopmental deficits [2]; however, significant neurocognitive difficulties have been documented among individuals who have been prenatally exposed to alcohol but do not meet full criteria for FAS [3•]. These individuals are described as having partial FAS (pFAS), alcohol-related neurodevelopmental disorder (ARND), or alcohol-related birth defects (ARBDs), according to the diagnostic categories proposed by the Institute of Medicine [4]. Despite public awareness campaigns over the years, PAE remains a significant public health concern, affecting up to $5 \%$ of children in the USA and in some Western European countries [5], with the highest prevalence rates being described in certain regions of Africa [6].

Adverse effects of PAE vary with the amount and pattern of alcohol consumed, although the severity of effects can vary substantially, even among individuals with similar levels of exposure. Thus, a number of environmental mechanisms likely contribute to varying patterns of deficits. Moreover, both genetic and epigenetic mechanisms may play a role in mediating the broad range of effects described in individuals with $\operatorname{PAE}[7 \cdot]$.

Given the neurocognitive problems associated with PAE, it is not surprising that psychosocial dysfunction has been consistently noted in the literature. Longitudinal studies suggest that individuals with PAE are at an increased risk for adverse long-term outcomes, including mental health problems and poor social adjustment [8]. This review summarizes the current literature on the underlying mechanisms of PAE vulnerability, including epigenetic, genetic, and environmental risk factors that predispose individuals with PAE to psychiatric illness. The studies cited are from animal and human research and include a developmental perspective. 


\section{Epigenetic Mechanisms}

Investigation of possible epigenetic mechanisms as mediators of alcohol's adverse effects is contributing to a better understanding of the negative outcomes related to PAE. Epigenetic mechanisms contribute to differential gene expression through chemical modification of DNA. One example of an epigenetic change is DNA methylation - the addition of a methyl group, or a "chemical cap," to part of the DNA molecule, which prevents certain genes from being expressed. DNA methylation has been studied in many epigenetic studies because it is the most accessible epigenetic marker. During prenatal and early postnatal development, the epigenome is very susceptible to environmental agents such as diet, maternal stress, alcohol, and other drugs. Three periods of early development - preconception, preimplantation (the first 2 weeks of gestation), and gastrulation (3-8 weeks of gestation)-have been demonstrated to be particularly sensitive to the teratogenic effects of alcohol.

Preconception effects of both paternal and maternal alcohol consumption are reported to include low birth weight, increased physical malformations, and problems in learning and mobility in animals $[9,10]$. These findings point to the possible contribution of the father's drinking to developmental outcome, which is a question often asked of alcohol researchers.

Research in mice suggests that in utero alcohol exposure during the preimplantation period manifests in adverse outcomes toward the extreme end of the FASD continuum [9]. For example, in one study, intraperitoneal administration of $5.8 \mathrm{~g} / \mathrm{kg}$ of ethanol during the preimplantation period resulted in growth retardation and severe physical malformations in almost all viable embryos studied [11]. In contrast, in vitro preparations do not have the same result, suggesting that the teratogenic consequences of ethanol exposure during the preimplantation period partly depend on an interaction with the maternal system (e.g., through production of toxic metabolites, such as acetaldehyde).

The central nervous system seems to be most sensitive to alcohol exposure throughout the period of gastrulation. Alcohol exposure during gastrulation has been linked to pronounced social deficits and enhanced anxiety in social circumstances in rats [12]. Findings in humans reveal increases in reactivity of the stress hormone cortisol, an elevated heart rate, and increased negative affect in 5- to 7-month-old infants in response to a social emotional stressor [13]. These findings imply that exposure to alcohol during early gastrulation may alter gene expression patterns in basic cell-signaling pathways involved in limbic/neuroendocrine development, resulting in reprogramming of the hypothalamic-pituitary-adrenal (HPA) axis and stress-related autonomic and behavioral reactivity $[7 \bullet, 14]$. The results have implications for the mental health outcomes of individuals with prenatal alcohol exposure, which are described in this paper.

\section{Genetic and Environmental Factors}

Rodent models have been used most often in studying the complex gene-environment interactions that alter gene expression patterns during development. The influence of maternal genotype on the metabolism of alcohol during pregnancy suggests a genetic basis for some of the effects of alcohol on the developing fetus. For example, there are alcoholsusceptible and alcohol-unaffected inbred mouse strains, which produce variable outcomes in their offspring despite similar levels of exposure to alcohol [15]. The maternal effect on susceptibility to the syndrome is related to maternal blood alcohol levels (BALs), which are postulated to be determined by both the amount of alcohol consumed and maternal genotype. Polymorphisms in the genetic encoding of the enzyme alcohol dehydrogenase $(\mathrm{ADH})$, which catalyzes the oxidation of ethanol to acetaldehyde, may play a role in the teratogenic effects of PAE. Moreover, there is an inverse relationship between maternal ADH activity and maternal BALs and consequent abnormalities. Recent research with animals confirms that two closely related C57BL/6 mouse lines (B6J and B6N) result in differences in genetic susceptibility of the developing face and brain to alcohol-related anomalies [16].

Similarly, studies in humans have found that that the presence of variants of the $A D H 1 B$ gene, which are associated with faster and more efficient ethanol clearance, may provide some protection from the adverse effects of alcohol exposure, although the amount of variance accounted for is small [17, 18•]. Such reasoning predicts that two women with differing alcohol-related genotypes, drinking the same amount of alcohol, will have offspring with varying degrees of the disorder.

The influence of genetic variation may explain some of the conflicting reports on the effect of moderate PAE on developmental outcomes in humans. Epidemiological research on the effects of PAE has generated contrasting and somewhat paradoxical findings. In recent studies in Britain, Australia, and Denmark, light to moderate alcohol consumption and occasional binge drinking have been associated with modest improvements in socioemotional and attentional outcomes [19-21]. These findings have been sensationalized in the press and have led to confusing messages to women about the safety of drinking during pregnancy. In a recent systematic review, O'Leary and Bower [22 $2^{\bullet}$ concluded that the results of studies showing significant protective effects from low to moderate levels of PAE could be explained by bias due to confounding by environmental variables, misclassification of exposure levels, or poorly predictive outcome measures. Importantly, these studies are confounded by environmental factors- such as older maternal age, stable marital status, higher levels of education, fewer mental health problems, and higher socioeconomic status - that are associated with light to moderate drinking. In contrast, associations between genotype and outcome are unlikely to be confounded by lifestyle factors, and so 
associations between child outcomes and maternal genotype may shed light on the effects of maternal alcohol consumption on child development. In a recent study using Mendelian randomization, Zuccolo and associates [18•] investigated relations between child outcomes (IQ and school achievement) and a maternal variant in the $A D H 1 B$ gene, which is associated with rapid alcohol metabolism. As noted above, this gene appears to have some protective factors in that it is linked to peak BALs, and carriers (fast metabolizers) are more likely to consume less alcohol before pregnancy and to abstain from alcohol in the first trimester, and are less likely to binge drink during pregnancy. The findings were that although outcomes were confounded with environmental factors, offspring of women whose genotype was related to a propensity to drink less and who metabolized ethanol faster performed better on achievement tests. Like the results from animal studies, these findings indicate that outcomes are highly dependent on multiple variables (including genetic variation), and caution is needed with regard to counseling women about the safety of drinking during pregnancy.

\section{Animal Models of Mental Health Problems}

Perhaps the most elegant work examining the mental health outcomes of PAE, using animal models, comes from the work of Weinberg and associates $[7 \bullet, 14]$. Negative developmental outcomes observed in human FASD have primarily been replicated using rodent models. These include growth retardation, physical anomalies, central nervous system abnormalities, and a wide range of behavioral and cognitive deficits, such as problems in learning and memory, poor self regulation, high activity levels, hyper-responsiveness to environmental stressors, and difficulty understanding environmental cues. Importantly for the current review, animal models of depression and anxiety have been described. Studies suggest that long-term alterations in HPA regulation and responsiveness in offspring with PAE, particularly under conditions of stress, reflect increased HPA tone throughout life, with subsequent vulnerability to psychiatric illness [14].

Confirmatory studies show that animals with PAE exhibit altered HPA responsiveness in challenge tests similar to those used to assess depression in humans [14]. Moreover, they exhibit behavioral characteristics of depression in tests designed to examine depressive-like behavior. These include tests of exploratory behavior, social interaction, reward seeking, sexual behavior, novelty seeking, and behavioral "despair," such as learned helplessness. Importantly, these tests are conducted in conjunction with stress exposure in order to mimic the diathesis stress model of mental illness proposed for humans.

Findings from Weinberg and associates $[7 \bullet, 14]$ reveal that stress induction methods alter behavioral and endocrine measures of activity in a manner parallel to those observed in depressive/anxiety-like disorders in humans, and that exposure to PAE results in an increase in vulnerability to depression and anxiety in a gender-specific way. Under stress conditions, males with PAE show greater anxiety, impaired pleasure seeking, an increase in hyperactivity, and alterations in social behavior, compared with unexposed control males. In contrast, despite showing anxiety and altered social interactions, females show greater levels of behavioral despair.

Animal models also have been used to explain the relation between PAE and later substance use disorders (SUDs) [23]. Under ad libitum conditions, rodent models of PAE show an enhanced preference for alcohol and increased alcohol consumption [24]. The underlying mechanisms of increased risk for SUDs appear to be related to long-lasting alterations in both the HPA and dopaminergic (DA) systems, as well as HPA-DA interactions in PAE males and females, with the PAE animals showing widespread changes in basal regulation of the central stress circuitry. Both the HPA and DA systems are important for resilience against addiction, which appears to be disrupted by PAE [25].

Animal models using higher-order primates (rhesus monkeys) mirror those conducted with rodents and have demonstrated that, under conditions of prenatal stress and alcohol exposure, outcomes include lower birth weights, increased stereotypical behavior, reduced attention span, decreased exploration and play behavior, and increased activity levels early in life [26]. Importantly, prenatally stressed adolescent monkeys with PAE exhibit an increase in alcohol preference over time, suggesting a vulnerability to SUDs similar to that found in humans.

In summary, the literature suggests complex etiologies for the negative psychiatric outcomes observed in individuals with PAE. As noted by Kelly et al. [27] in their review of the animal and human research, there are changes in social behavior induced by alcohol exposure, and these changes can be observed early in life, affecting the foundations of social behavior throughout life and ultimately leading to an increase in the rates of depression, suicide, and other maladaptive behaviors shown in individuals with PAE.

\section{Human Studies of Psychiatric Illness}

\section{Infancy and Early Childhood}

At birth, there are signs of central nervous system dysfunction in infants born to mothers who report consuming large quantities of alcohol during pregnancy. These include reports of jitteriness, irritability, autonomic instability, increased levels of activity, and disturbances in sleep patterns [28]. The significance of these early neurobehavioral effects is apparent in the impact they may have on early mother-child interactions. 
Thus, the effects of alterations in child behavior on the mother-child relationship may be one of the most significant results of PAE.

To examine the complex developmental process resulting in the emergence of childhood depression, we examined the associations among maternal prenatal and postnatal alcohol use, maternal depression, mother-child interactions, and child depressive symptoms in the 6-year-old offspring of a relatively low-risk group of middle-class social drinkers. We found that early irritability and poor mother-child interactions in the more heavily exposed infants at 1 year of age predicted higher levels of depression at 6 years of age, particularly for girls [8]. These findings emerged even though the mothers had not been identified as high-risk drinkers and the children were functioning within the high average range of intelligence. Interestingly, PAE and maternal depression had an additive predictive effect, contributing independently and significantly to prediction of childhood depression. These findings could not be explained by the mother's past use of other substances (cocaine, nicotine, marijuana, caffeine) or smoking during pregnancy, current drinking practices, or current mother-child interaction patterns.

The results from a study of a community sample of 323 participants, examining the impact of PAE, maternal depressive symptoms, and emotional support from the father on infant irritability at 5 and 17 months of age, confirmed our previous findings and identified father emotional support as an additional predictive factor [29]. Fetal alcohol exposure, low emotional support from the father, and maternal depressive symptoms were associated with increased infant irritability at 5 months. However, at the age of 17 months, only a main direct effect of PAE on irritability was found. These results suggest that PAE involves an enduring vulnerability, which has a more lasting influence on temperamental outcomes than factors such as maternal depressive symptoms and emotional support from significant others.

In order to better understand the association between PAE and socioemotional problems in high-risk children, we conducted a series of studies of children with higher levels of cumulative risk than our first sample [8]. These alcoholexposed children were from lower socioeconomic backgrounds and were living with their biological mothers, the majority of whom were single parents. Strikingly, we found that the majority of children $(80 \%)$ in the moderate-heavy alcohol exposure group displayed insecure attachment behaviors, while only $36 \%$ of children in the abstinent-light alcohol exposure group were insecurely attached. Further analyses provided insight beyond documentation of the direct relationship between PAE and the quality of attachment. Prenatal exposure was related to temperamental differences in the child (similar to those found in the middle-class sample), and these temperamental differences affected the mother's ability to relate to her child emotionally, suggesting that the quality of the mother-child relationship can exacerbate the effects of PAE on attachment security in the preschool years.

Additional research with this higher-risk sample has replicated and extended the results of greater self-reported depressive symptoms among children more heavily exposed to alcohol in utero [30]. Like their middle-class counterparts, mothers living in poverty had children evidencing higher levels of depressive symptoms. Moreover, PAE appeared to predispose children to show more negative affect when interacting with their mothers, and the mothers of these children were less emotionally connected to their offspring. These relations persisted even after adjustment for prenatal and current use of other drugs. Importantly, a direct relation between prenatal alcohol exposure and child depressive symptoms was found. This result, in combination with the finding that more heavily exposed children also had smaller head circumferences, is consistent with neuroanatomical findings suggesting that PAE is associated with smaller brain volume and with possible damage to the medial prefrontal cortex and closely related areas implicated in the regulation of mood [31].

Although most studies showing an association between maternal alcohol misuse and psychiatric symptoms in children have generally been interpreted as reflecting the impact of the postnatal environment-and especially the effects of living with an alcoholic mother-we believe that our results show that PAE can also act as a significant risk factor in the emergence of early-onset psychopathology. This risk appears to be conveyed through the mother's own genetic vulnerability and mental health, the response to primary temperamental deficits resulting from the child's exposure to alcohol in utero, and the direct effects of alcohol on brain development itself.

\section{Middle Childhood}

\section{Internalizing Symptoms: Depression, Anxiety}

A few studies are notable for examining psychopathology in children with FASD in middle childhood. Studies of relatively small samples have suggested that psychiatric problems are more common in children with PAE than was previously thought $[8,32]$.

In a large sample of 130 non-clinic children, using the National Institute for Mental Health Computerized Diagnostic Interview Schedule for Children-Fourth Edition (NIMH CDISC-IV [33], we found that mood disorder symptoms were significantly greater in children with PAE than in those without exposure and were mediated by environmental risk variables [34]. Similarly, a large prospective sample of children exposed to low to moderate levels of alcohol before birth revealed an association between higher levels of PAE and higher rates of internalizing problems (depression/anxiety) at the age of 10 years, the effect of which was attenuated by maternal risk factors [35]. 
The link between PAE and anxiety has been less well studied than the links between PAE and mood disorders and disorders of impulse control; however, our own research on infant attachment behavior has demonstrated a higher prevalence of attachment insecurity in children with moderate to high PAE than in those with little or no exposure. Similarly, examining anxiety in a large sample of latency-aged children, we found an association between symptoms of anxiety and PAE [34]. The risk associated with anxiety symptoms underscores the idea that children with PAE are prime candidates for early interventions focusing on reducing anxiety and increasing coping skills, through facilitation of the mother-child relationship.

Another well-controlled study of young children, using a prospective longitudinal approach, deserves mention. Sayal and associates [36] sampled the drinking patterns of 12,678 pregnant women during the first 18 weeks of gestation and measured the mental health outcomes of their offspring at 4 and 8 years of age. The authors found that consumption of less than one drink per week during the first trimester was associated with clinically significant mental health problems, particularly in girls. This study emphasizes the potential dangerous effects of even small levels of alcohol consumption during pregnancy on developmental outcomes. These findings are consistent with our own, suggesting higher levels of vulnerability to internalizing disorders in young females, and are consistent with demonstrated gender differences in the effects of PAE on responsiveness to stress, anxiety, and depression in animal studies [14].

\section{Externalizing Symptoms: Attention Deficit Hyperactivity Disorder, Oppositional Defiant Disorder, and Conduct Disorder}

The most commonly reported findings suggest that children with PAE exhibit an increased prevalence of externalizing and disruptive behavior diagnoses. For example, a recent study examining the psychiatric conditions of a clinically referred sample revealed that $97 \%$ of the PAE children met criteria for at least one Axis I diagnosis in the Diagnostic and Statistical Manual of Mental Disorders, Fourth Edition (DSM-IV) [28]. Of that number, $28 \%$ met criteria for a mood or anxiety disorder, but the majority met criteria for externalizing disorders such as attention-deficit/hyperactivity disorder (ADHD), oppositional defiant disorder (ODD), or conduct disorder (CD). Moreover, recent findings from the same lab revealed that individuals with PAE were more likely to have a greater number of comorbid psychiatric illnesses than unexposed control subjects, and that the presence of a diagnosis of ADHD conferred the greatest degree of comorbid psychiatric risk [37].

We found similar significant differences between children with and without PAE in the disruptive disorders categories
[34]. ADHD, ODD, and CD were all positively predicted by PAE, after adjustment for the contributions of IQ, social skills deficits, and home placement. These problems in conduct are similar to those found in a study examining data from the National Longitudinal Survey of Youth, which revealed that there was an association between PAE and conduct problems and that this was independent of confounding genetic effects, other in utero drug exposure, or environmental effects [38].

Although attention deficits among children with FASDs are well documented in the literature and corroborate the widespread presence of these problems in children with PAE [39-41], investigators have suggested that while they may share common features, the inattention and hyperactivity associated with PAE denote a particular clinical subtype with an earlier onset, a different clinical and neuropsychological presentation, and probably a differential medication response than idiopathic ADHD [3•, 39, 42]. Significantly, results reported from three large population-based pregnancy offspring cohorts within the Nordic Network on ADHD found that PAE was not related to risk for ADHD after adjustment for covariates [43]. In summary, although alcohol-exposed children may share some behavioral characteristics with children diagnosed with ADHD, current reports on this particular behavioral outcome of PAE are not conclusive.

\section{Adolescents and Young Adults}

The prevalence of mental health problems in adolescents and young adults has been reported to be higher than $90 \%$ [44]. Alarmingly, the psychiatric problems of this population are underscored by the high degree of reported suicidal risk [45]. In our own lab, the rate of serious suicide attempts in adolescents with PAE was $5 \frac{1}{2}$ times the national average (O'Connor et. al; Suicide risk in adolescents with fetal alcohol spectrum disorders, 2012, unpublished).

As described in samples of younger children, problems in impulse control and conduct continue to be highly associated with PAE during the adolescent period. In an investigation of 1,252 17-year-old adolescents from the Minnesota Twin Family Study, PAE was associated with higher levels of conduct disorder symptoms in offspring, even after adjustment for the effects of parental externalizing disorders (illicit substance use disorders, alcohol dependence, and antisocial/behavioral disorders), prenatal nicotine exposure, monozygosity, gestational age, and birth weight [46].

An analyses of the 21-year outcomes of a Seattle longitudinal study comparing subjects who were exposed prenatally to a "binge" pattern of alcohol exposure ( $>5$ drinks on at least one occasion) with those who had low or moderate levels of PAE revealed that young adults exposed to a binge pattern of maternal alcohol use reported significantly higher overall levels of psychiatric symptoms. Follow-up of this sample at the age of 25 years revealed that the odds of the appearance of 
passive-aggressive and antisocial personality disorders were twice as high in adults exposed to one or more binge episodes as in those who were exposed to low to moderate levels [47].

Looking more specifically at DSM-IV Axis I disorders, in an older study, the authors reported that $92 \%$ of their sample of alcohol-exposed adults met criteria for an Axis I diagnosis [48]. Sixty-eight percent met criteria for a mood disorder, including major depression (44\%), bipolar I disorder (20\%), or dysthymia (4\%). Furthermore, $20 \%$ met criteria for an anxiety disorder and $40 \%$ met criteria for a psychotic disorder. Gender differences were noted in that females were found to have higher prevalence rates of depression (50\%) and anxiety (50\%) than males (40\% and $0 \%$, respectively). In a Canadian study of 24 adults with PAE participating in an intervention program, Denys et al. [49] found that $83 \%$ had at least one mental health diagnosis, with the most common being depression, anxiety, or ADHD.

As they mature, individuals with PAE exhibit problems with the misuse of alcohol and other drugs often associated with psychiatric symptoms. Two well-designed prospective studies have shed light on this relationship [50-52]. Early work from the Seattle Longitudinal Prospective Study on Alcohol and Pregnancy revealed a relation between exposure and early experiences with alcohol among young adolescents at 14 years of age [50]. The relationship between PAE, heavy drinking, and alcohol-related problems persisted into early adulthood [51].

In a similar prospective study, the association between maternal alcohol use during pregnancy and early drinking was examined in 4,363 14-year-old adolescents taking part in the Mater University of Queensland Study of Pregnancy and Its Outcomes (MUSP) [52]. After adjustment for other factors, adolescents whose mothers consumed $\geq 3$ drinks per drinking occasion during pregnancy were at increased risk for drinking more alcohol in a binge pattern than those whose mothers consumed less alcohol. A follow-up study of this cohort at the age of 21 years revealed a strong relationship between maternal binge drinking in early pregnancy and alcohol use disorders in the adult offspring.

\section{Inclusion of Neurobehavioral Disorder Associated with Prenatal Alcohol Exposure in the DSM-5}

The probability of obtaining an early diagnosis of a psychiatric problem in individuals with PAE is hindered by a general lack of clinical awareness. The Diagnostic and Statistical Manual of Mental Disorders, Fifth Edition (DSM-5) [53] partially addresses the need for mental health diagnostics for this population by including Neurobehavioral Disorder Associated with Prenatal Alcohol Exposure (ND PAE) in Section III, an area devoted to conditions that require further research before consideration as formal disorders. ND PAE is defined in the DSM- 5 by exposure to alcohol during gestation, neurocognitive impairment, impairment in self-regulation, and deficits in adaptive functioning that are apparent before the age of 18 years and that contribute to clinically significant distress or impairment in social, occupational, or other important areas of functioning. The current guidelines allow ND PAE to be diagnosed in the presence or absence of the physical effects of PAE. The rationale for including ND PAE in conditions requiring further study acknowledges the concerns raised by parents, researchers, clinicians, and state and national medical and advocacy organizations about the problems associated with its exclusion from the DSM. Of particular concern, individuals impacted by PAE are significantly overrepresented yet under-recognized in the child welfare system, juvenile detention and correctional facilities, and outpatient and inpatient psychiatric settings $[8,54,55]$. Recognizing this problem, the American Bar Association put forth a resolution that urges attorneys and judges; state, local, and specialty bar associations; and law school clinical programs to identify and respond effectively to FASD in children and adults, through training to enhance awareness of FASD and its impact on individuals in the child welfare, juvenile justice, and adult criminal justice systems [56]. Although these initiatives and ND PAE's provisional inclusion in the DSM are encouraging, such inclusion in Section III, instead of in the "Neurodevelopmental Disorders of Childhood" section, speaks to the disorder's ongoing marginal status in psychiatric practice.

\section{Conclusions}

The findings from this review reveal that mental health problems are prominent in populations of individuals with PAE and occur throughout the lifespan. Also evident are the findings that other influences can impact developmental trajectories. These influences include (but are not limited to) genetic and epigenetic phenomena, exposure to other teratogens during pregnancy, environmental stressors, socioeconomic status, and problems in parenting, including maternal mental health, and represent a continuum of developmental risk.

Despite the evidence of a significant association between alcohol exposure in utero and psychiatric risk, experience suggests that exposure, and even FAS, are infrequently identified by mental health practitioners as relevant contributing factors. This omission is unfortunate, given the observations of treatment resistance to medications and psychosocial therapies, as well as the frequent need for specialized educational services, in this population. Research on the psychiatric disabilities suffered by individuals with PAE throughout the lifespan highlight the need for training of mental health professionals in the identification of people with FASD and the 
provision of specific treatments to address the unique features of this developmental disability. Failure to recognize the broad and unique needs of these individuals and their families can lead to multiple treatment failures, consequent worsening of symptoms, and high personal and societal costs.

Acknowledgments The writing of this manuscript was supported, in part, by funding from the Centers for Disease Control and Prevention (grant number UDD000041). The contents do not necessarily represent the positions or policies of the Centers for Disease Control and Prevention, and endorsement by the Federal Government should not be assumed.

\section{Compliance with Ethics Guidelines}

Conflict of Interest Mary J. O'Connor declares that she has no conflict of interest.

Human and Animal Rights and Informed Consent This article does not contain any studies with human or animal subjects performed by any of the authors.

\section{References}

Papers of particular interest, published recently, have been highlighted as:

- Of importance

1. Warren KR, Hewitt BG, Thomas JD. Fetal alcohol spectrum disorders: research challenges and opportunities. Alcohol Res Health. 2011;34:4-14. Overview of diagnosis and developmental deficits associated with PAE. Includes discussion of possible epigenetic, genetic, and environmental mechanisms.

2. Jones KL, Smith DW. Recognition of the fetal alcohol syndrome in early infancy. Lancet. 1973;2:999-1001.

3. Mattson SN, Roesch SC, Glass L, Deweese BN, Coles CD, Kable JA, et al. Further development of a neurobehavioral profile of fetal alcohol spectrum disorders. Alcohol Clin Exp Res. 2013;37:51728. Identifies a neurobehavioral profile distinguishing individuals with PAE from typically developing children and those with ADHD.

4. Stratton K, Howe C, Battaglia F, editors. Fetal alcohol syndrome: diagnosis, epidemiology, prevention, and treatment. Washington, DC: Institute of Medicine, National Academy Press; 1996.

5. May PA, Gossage JP, Kalberg WO, Robinson LK, Buckley D, Manning M, et al. Prevalence and epidemiologic characteristics of FASD from various research methods with an emphasis on recent in-school studies. Dev Dis Res Rev. 2009;15:176-92.

6. May PA, Blankenship J, Marais AS, Gossage JP, Kalberg WO, Barnard R, et al. Approaching the prevalence of the full spectrum of fetal alcohol spectrum disorders in a South African populationbased study. Alcohol Clin Exp Res. 2013;37:818-30.

7. Kobor MS, Weinberg J. Focus on epigenetics and fetal alcohol spectrum disorders. Alcohol Res Health. 2011;34:29-37. Focus on possible epigenetic mechanisms that alter outcomes of individuals with PAE.

8. O'Connor MJ, Paley B. Psychiatric conditions associated with prenatal alcohol exposure. Dev Disabil Res Rev. 2009;15:225-34.

9. Haycock PC. Fetal alcohol spectrum disorders: the epigenetic perspective. Biol Reprod. 2009;81:607-17.
10. Kaminen-Ahola N, Ahola A, Flatscher-Bader T, Wilkins SJ, Anderson GJ, Whitelaw E, et al. Postnatal growth restriction and gene expression changes in a mouse model of fetal alcohol syndrome. Birth Defects Res A Clin Mol Teratol. 2010;88(10):818-26.

11. Padmanabhan R, Hameed MS. Effects of acute doses of ethanol administered at pre-implantation stages on fetal development in the mouse. Drug Alcohol Depend. 1988;22:91-100.

12. Mooney SM, Varlinskaya EI. Acute prenatal exposure to ethanol and social behavior: effect of age, sex, and timing of exposure. Behav Brain Res. 2011;216:358-64.

13. Haley DW, Handmaker NS, Lowe J. Infant stress reactivity and prenatal alcohol exposure. Alcohol Clin Exp Res. 2006;30:205564.

14. Hellemans KGC, Silwowkka JA, Verma P, Yoon VE, Yu W, Weinberg J. Prenatal alcohol exposure: fetal programming and later life vulnerability to stress, depression and anxiety disorders. Neurosci Biobehav Rev. 2010;34:791-807.

15. Downing C, Balderrama-Durbin C, Broncucia H, Gilliam D, Johnson TE. Ethanol teratogenesis in five inbred strains of mice. Alcohol Clin Exp Res. 2009;33:1238-45.

16. Ml G, Singh AV, Zhang Y, Nemeth KA, Sulik KK, Knudsen TB. Reprogramming of genetic networks during initiation of the fetal alcohol syndrome. Dev Dyn. 2007;236:613-31.

17. Jacobson SW, Carr LG, Croxford J, Sokol RJ, Li TK, Jacobson JL. Protective effects of the alcohol dehydrogenase- $A D H 1 B$ allele in children exposed to alcohol during pregnancy. J Pediatr. 2006;148: 30-7.

18. Zuccolo L, Lewis SJ, Smith GD, et al. Prenatal alcohol exposure and offspring cognition and school performance. A 'Mendelian randomization' natural experiment. Int J Epidemiol. 2013;42: 1358-70. Experimentally tests the hypothesis that effects from $P A E$ are related to genetic mechanisms irrespective of environmental variables.

19. Kelly YJ, Sacker A, Gray R, Kelly J, Wolke D, Head J, et al. Light drinking during pregnancy: still no increased risk for socioemotional difficulties or cognitive deficits at 5 years of age? J Epidemiol Community Health. 2012;66:41-8.

20. Robinson M, Oddy WH, McLean NJ, Jacoby P, Pennell CE, de Klerk NH, et al. Low-moderate prenatal alcohol exposure and risk to child behavioural development: a prospective cohort study. BJOG. 2010;117:1139-50.

21. Underberg M, Kesmodel US, Landro NJ, Bakketeig L, Grove J, Wimberly T, et al. The effects of low to moderate alcohol consumption and binge drinking in early pregnancy on selective and sustained attention in 5-year-old children. BJOG. 2012;119:121121.

22. O'Leary CM, Bower C. Guidelines for pregnancy: what's an acceptable risk, and how is the evidence (finally) shaping up? Drug Alcohol Rev. 2012;31:170-83. A critical review of studies purporting to demonstrate the beneficial effects of moderate prenatal alcohol consumption on developmental outcome.

23. Uban KA, Comeau WL, Ellis LA, Galea LAM, Weinberg J. Basal regulation of HPA and dopamine systems is altered differentially in males and females by prenatal alcohol exposure and chronic variable stress. Psychoneurendocrinology. 2013;38:1953-66.

24. Chotro MG, Arias C, Laviola G. Increased ethanol intake after prenatal ethanol exposure: studies with animals. Neurosci Biobehav Rev. 2007;31:181-91.

25. Sinha R. Stress and addiction: a dynamic interplay of genes, environment, and drug intake. Biol Psychiatry. 2009;66:100-1.

26. Schneider ML, Moor CF, Adkins MM. The effects of prenatal alcohol exposure on behavior: rodent and primate studies. Neuropsychol Rev. 2011;21:186-203.

27. Kelly SJ, Day N, Streissguth AP. Effects of prenatal alcohol exposure on social behavior in humans and other species. Neurotoxicol Teratol. 2002;22:143-9. 
28. Fryer SL, McGee CL, Matt GE, Riley EP, Mattson SN. Evaluation of psychopathological conditions in children with heavy prenatal alcohol exposure. Pediatrics. 2007;119:733-41.

29. Lemola S, Stadlmayr W, Crob A. Infant irritability: the impact of fetal alcohol exposure, maternal depressive symptoms, and low emotional support from the husband. Infant Ment Health J. 2009;30:57-81.

30. O'Connor MJ, Paley B. The relationship of prenatal alcohol exposure and the postnatal environment to child depressive symptoms. J Pediatr Psychol. 2006;31:50-64.

31. Drevets WC, Price AE, Furye ML. Brain structural and functional abnormalities in mood disorders: implications for neurocircuitry models of depression. Brain Struct Funct. 2008;213:93-118.

32. Pei J, Denys K, Hughes J, Rasmussen C. Mental health issues in fetal alcohol spectrum disorder. J Ment Health. 2011;20(5):438-48.

33. Shaffer D, Fisher P, Lucas CP, Dulcan MK, Schwab-Stone ME. NIMH Diagnostic Interview Schedule for Children Version IV (NIMH DISC-IV): description, differences from previous versions, and reliability of some common diagnoses. J Am Acad Child Adolesc Psychiatry. 2000;39:28-38.

34. Walthall JC, O'Connor MJ, Paley B. A comparison of psychopathology in children with and without prenatal alcohol exposure. Ment Health Asp Dev Disabil. 2008;11:69-78.

35. Leech SL, Larkby CA, Day R, Day NL. Predictors and correlates of high levels of depression and anxiety symptoms among children at age 10. J Am Acad Child Adolesc Psychiatry. 2006;45:223-30.

36. Sayal K, Heron J, Golding J, Emond A. Prenatal alcohol exposure and gender differences in childhood mental health problems: a longitudinal population-based study. Pediatrics. 2007;119:426-34.

37. Ware AL, O'Brien JW, Crocker N, et al. The effects of prenatal alcohol exposure and attention-deficit/hyperactivity disorder on psychopathology and behavior. Alcohol Clin Exp Res. 2013;37(3):507-16.

38. D'Onofrio BM, Van Hulle CA, Waldman ID, Rodgers JL, Rathouz PJ, Lahey BB. Causal inferences regarding prenatal alcohol exposure and childhood externalizing problems. Arch Gen Psychiatry. 2007;6:1296-304.

39. O'Malley KD, Nanson J. Clinical implications of a link between fetal alcohol spectrum disorder and attention-deficit hyperactivity disorder. Can J Psychiatry. 2002;47:349-54.

40. Burd L, Klug MG, Martsolf JT, Kerbeshian J. Fetal alcohol syndrome: neuropsychiatric phenomics. Neurotoxicol Teratol. 2003;25:697-705.

41. Burd L, Carlson C, Kerbeshian J. Fetal alcohol spectrum disorders and mental illness. Int J Disabil Hum Dev. 2007;6:383-96.

42. Mattson SN, Crocker N, Nguyen TT. Fetal alcohol spectrum disorders: neuropsychological and behavioral features. Neuropsychol Rev. 2011;21:81-101.

43. Rodriguez A, Olsen J, Kotimaa AJ, et al. Is prenatal alcohol exposure related to inattention and hyperactivity symptoms in children? Disentangling the effects of social adversity. J Child Psychol Psychiatry. 2009;50:1073-83.

44. Streissguth AP, Barr HM, Kogan JA, Bookstein FL. Understanding the occurrence of secondary disabilities in clients with Fetal Alcohol Syndrome (FAS) and Fetal Alcohol Effects (FAE). Final Report to the Centers for Disease Control and Prevention (CDC), August. Seattle: University of Washington, Fetal Alcohol \& Drug Unit, Tech. Rep. No. 96-06; 1996.

45. Huggins JE, Grant T, O'Malley K, Streissguth AP. Suicide attempts among adults with fetal alcohol spectrum disorders: clinical considerations. Ment Health Asp Dev Disabil. 2008;11:33-41.

46. Disney ER, Lacono W, McGue M, Tully E, Legrand L. Strengthening the case: prenatal alcohol exposure is associated with increased risk for conduct disorder. Pediatrics. 2008;122:1225-30.

47. Barr HM, Bookstein FL, O'Malley KD, Connor PD, Huggins JE, Streissguth AP. Binge drinking during pregnancy as a predictor of psychiatric disorders on the Structured Clinical Interview for DSMIV in young adult offspring. Am J Psychiatry. 2006;163:1061-5.

48. Famy C, Streissguth AP, Unis AS. Mental illness in adults with fetal alcohol syndrome or fetal alcohol effects. Am J Psychiatry. 1998;155:552-4.

49. Denys K, Rasmussen C, Henneveld D. The effectiveness of a community-based intervention for parents with FASD. Community Ment Health J. 2009;64:23-34.

50. Baer JS, Barr HM, Bookstein FL, Sampson PD, Streissguth AP. Prenatal alcohol exposure and family history of alcoholism in the etiology of adolescent alcohol problems. J Stud Alcohol. 1998;59: 533-43.

51. Baer JS, Sampson PD, Barr HM, Connor PD, Streissguth AP. A 21year longitudinal analysis of the effects of prenatal alcohol exposure on young adult drinking. Arch Gen Psychiatry. 2003;60:377-85.

52. Alati R, Clavarino A, Najman JM, O'Callaghan M, Bor W, Mamun AA, et al. The developmental origin of adolescent alcohol use: findings from the Mater University Study of Pregnancy and Its Outcomes. Drug Alcohol Depend. 2008;98:136-43.

53. American Psychiatric Association. Diagnostic and statistical manual of mental disorders. 5th ed. Washington, DC: American Psychiatric Association; 2013.

54. O'Connor MJ, Laugeson EA, Mogil C, Lowe E, Welch-Torres $\mathrm{K}$, Keil V, et al. Translation of an evidence-based social skills intervention for children with prenatal alcohol exposure in a community mental health setting. Alcohol Clin Exp Res. 2012;36:141-52.

55. O'Connor MJ, McCracken JT, Best A. Under recognition of prenatal alcohol exposure in a child inpatient psychiatric setting. Ment Health Asp Dev Dis. 2006;9:105-9.

56. American Bar Association. Resolution on FASD, 2012. http:// www.americanbar.org/content/dam/aba/administrative/mental physical_disability/Resolution_112B.authcheckdam.pdf. Accessed 11 Feb 2014. 